\title{
Prediction and Survival Rate Prostate Cancer Patient using Artificial Neural Network
}

\author{
Sameer Dixit \\ Asst. Professor \\ Integral University, Lucknow
}

\author{
Shraddha Srivastava \\ HBTI, Kanpur
}

\author{
Kamalesh Chandra Maurya \\ Integral University, Lucknow
}

\begin{abstract}
Prostate cancer is that starts in the prostate gland. The prostate is a small, walnut sized structure that makes up part of a man's reproductive system. It wraps around the urethra, the tube that carries urine out of the body. Prostate cancer is the most common cause of death from cancer in men over age 75 . Prostate cancer is rarely found in men younger than 40.Current method of screening for prostate cancer carried out through blood test \& presence of high PSA lead to a high percentage of false positive result which can be reduced by employing intelligent Artificial Neural Networks. The main aim of our research paper and the parallel undertaking of its practical implementation is to develop a mathematical model to improve prostate cancer detection and staging system and finally to present a deploy ready marketable solution based on the model which can be installed across various screening, centers ,hospital and research organization.
\end{abstract}

Keywords

PSA, ANN, MLP

\section{INTRODUCTION}

(1) Why is the emphasis on prostate cancer?

American Cancer Society (ACS) estimates for 2005 include 232090 new cases of prostate cancer in the U.S.Year2005 estimates include 30350 deaths occurring from prostate cancer in the US alone, making it the second leading cause of cancer death in men. African-American men have about a 70 percent higher incidence rate of prostate cancer Caucasian men and nearly a two-higher mortality rate than Caucasian men.

(2) The Need for efficient prostate cancer screening and diagnostic methods

Physicians are relying on the results from the PSA blood test to diagnose prostate cancer. Prostate-specific antigen (PSA) is a protein produced by cells of the prostate gland. The PSA test measures the level of PSA in the blood .The U.S. Food and Drug Administration (FDA) has approved the use of the PSA test along with a digital rectal exam to help detect prostate cancer in men age 50 and older. The FDA has also approved the PSA test to monitor patients with a history of prostate cancer to see if the cancer has recurred (come back)Doctors' recommendations for PSA screening vary. The higher a man's PSA level, the more likely it is that cancer is present, but there are other possible reasons for an elevated PSA level. Doctors take several factors into account for men who have a rising PSA level after treatment for prostate cancer. The PSA test for screening has limitations and is still controversial. Researchers are studying ways to validate and improve the PSA test and to find other ways of detecting prostate cancer early. Hence the goal of this paper is to employ Intelligent Artificial Neural Networks to build a deploy ready marketable mathematical model to reduce the non-required trial and error method and expedite early cancer diagnosis and treatment.

\section{PSPCPANN}

\section{Project Overview}

The idea is to build model to improve prostate cancer detection and staging system. Here the basis is to add intelligence to Artificial Neural Networks and produce a deploy-ready mathematical model that revolves around the concept of ANN. The actual implementation of the model would require building a standalone software application which would be installed across screening centers, hospitals, and research institutions. The project is in its intermediate stage and the team is working on the development and implementation stage and to bring the product to the masses.C is the choice of programming language to be used for the reasons of its strong mathematical library and Matlab is used as the simulation software package to test our software runs. This research project is a long term initiative involving successive refinements to the first version of our model.

\section{Artificial Neural Network-}

An ANN is efficient information processing system which resembles in characteristics with a biological neural network. The biological neuron consists of three main parts.

Soma or cell body - where the cell nucleus is located.

Dendrites - where the nerve is connected to the cell body.

Axon - This carries the impulses of the neuron.

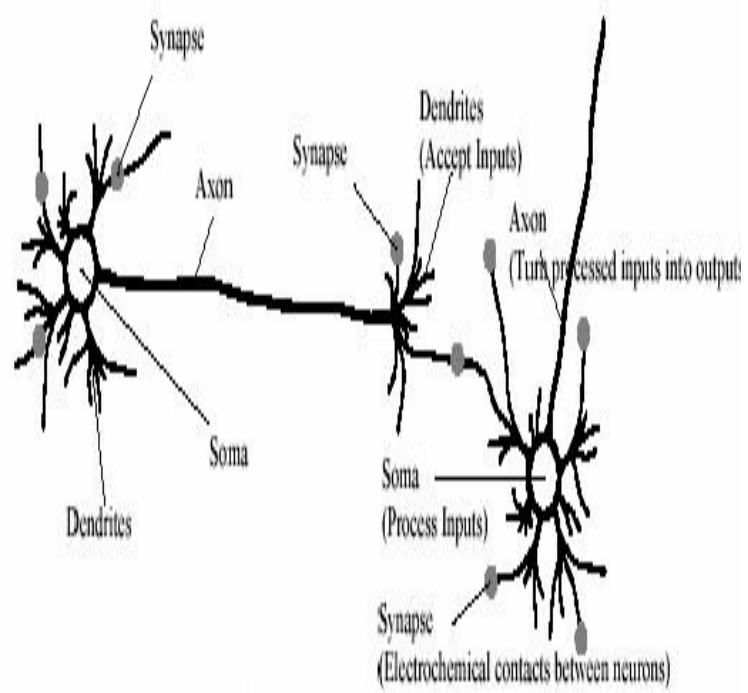




\section{Mathematical Representation of ANN-}

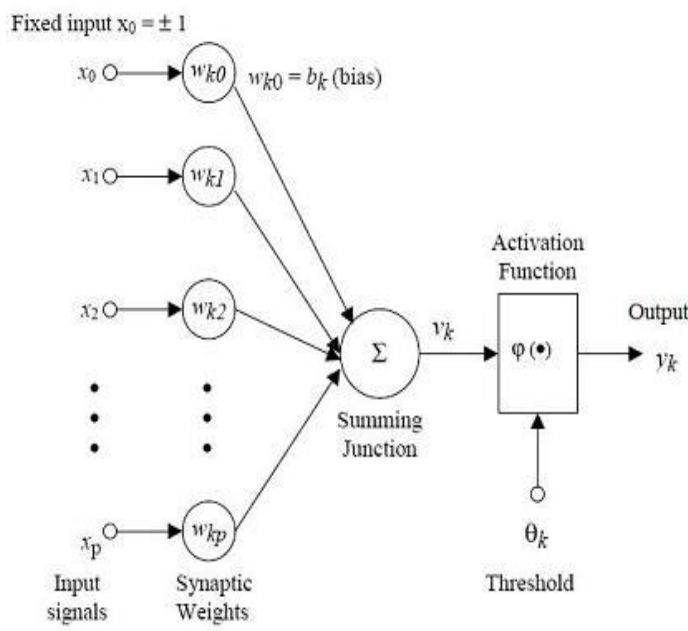

Yin $=b_{k}+\sum x_{\mathrm{p}} \mathrm{wk}_{\mathrm{p}}$

output $y=f($ Yin)

\section{Overview of prostate-}

Prostate cancer is cancer that starts in the prostate gland. The prostate is a small, walnut-sized structure that makes up part of a man's reproductive system. It wraps around the urethra, the tube that carries urine out of the body. Prostate cancer is the cancer beginning in the prostate which may remain in the gland or spread to other internal organ. Screening and Diagnosis are carried out through blood tests measuring the level of PSA and alternatively accompanied by a digital rectum exam(DRE).After initial diagnosis further staging system such on TNM may be undertaken top in point the location of cancer and degree of its spread into other regions.

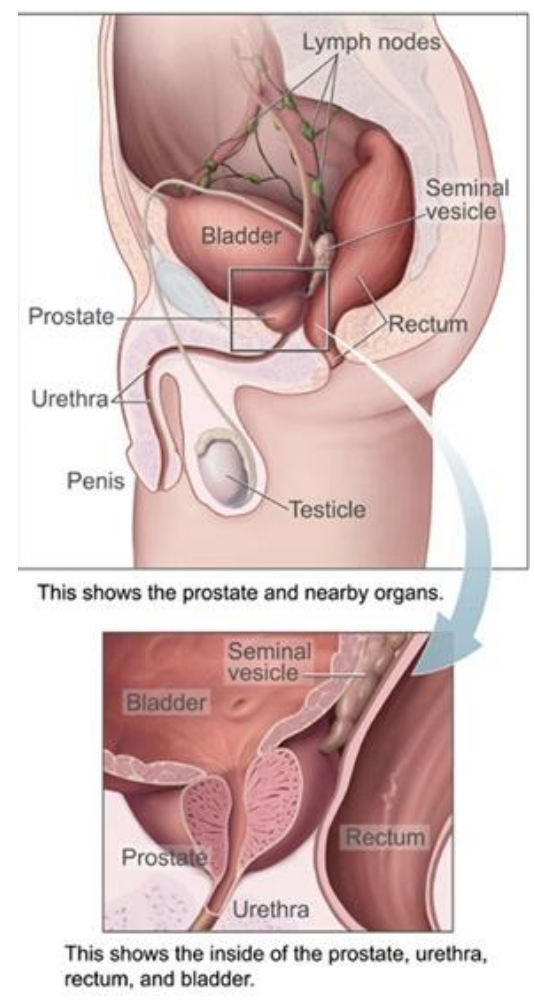

\section{ANN to prostate cancer analysis}

The current method of prostate cancer detection is finding the percentage of tPSA (total prostate specific antigen).However there is some drawback related to this approach. The first being that only cancer confirmed to the prostate gland can be detected and the second being that additional information such as degree of cancer spread to other internal organ requires more tissue samplings.

A possible solution to this problem may be found in finding the percentage of free prostate specific antigen (Fpsa) to suggest the degree of spread of prostate cancer to other parts. However both the above solution has the following drawback.

- They cannot provide specific diagnostic results on a person to person basis.

- $\quad$ There are unable to learn from existing diagnostic patterns and associate them with new input data from different patient failing under the same category based on

\section{MLP methodology}

Our neural network model used is a multilayer perceptron (MLP) network composed of one input layer with four primary preprocessed variables tPSA, fPSA, prostate volume, DRE.
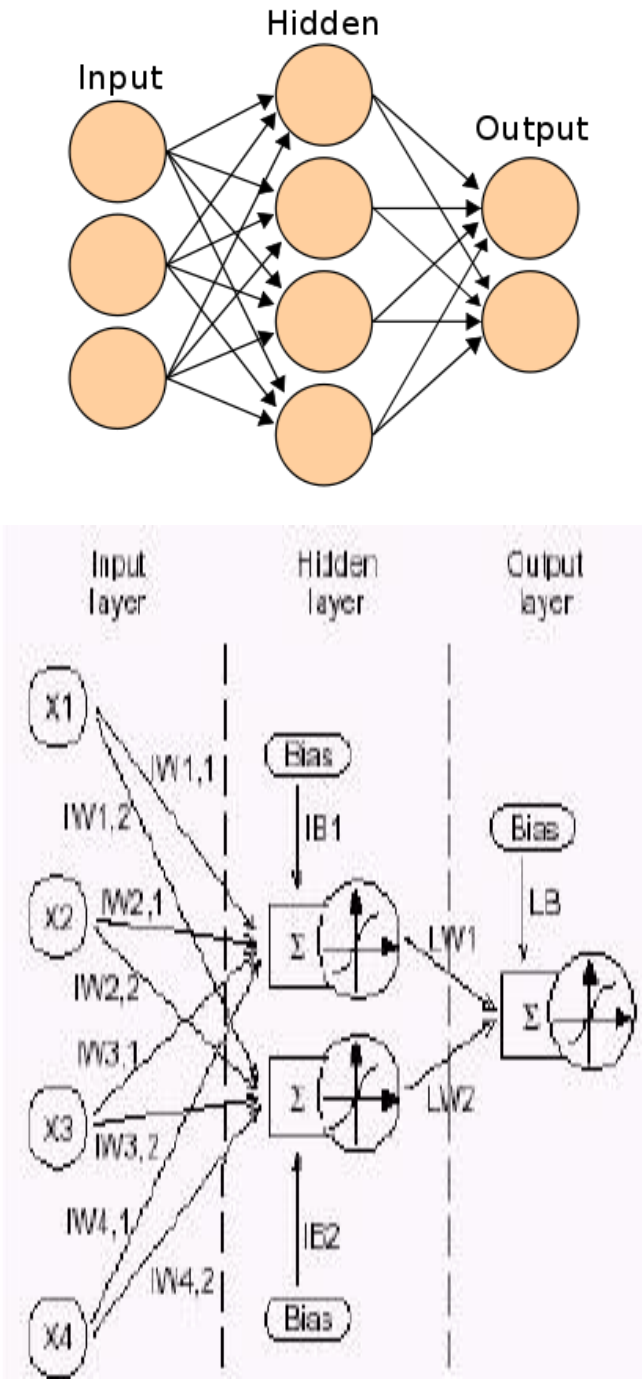
In which there is one hidden layer with two neurons and one output layer with one neuron giving the output value that is a measure of the probability of cancer. Here Activation function used in the hidden and output layers is Bipolar step function.

$$
\begin{aligned}
F(x)= & 1 \text { if } x>0 \\
& -1 \text { if } x<0
\end{aligned}
$$

Hence the output values are between -1 to 1 .

The formula for whole network thus become -

1. Activate input layer unit. For $i=1$ to 4

$$
\mathrm{Xi}=\mathrm{Si}
$$

2. Calculate net input to each unit

$$
\text { Zinj }=b j+\sum X i \text { Wij, } \quad \mathrm{j}=1 \text { to } 2
$$

3. Calculate output of each hidden unit -

$$
\mathrm{Zj}=\mathrm{f}(\text { Zinj) }
$$

4. Find the output of the net -

$$
\text { Yin }=\text { bo }+\sum \mathrm{Zj} \mathrm{LWj}
$$

$$
\mathrm{Y}=\mathrm{f}(\mathrm{Yin})
$$

After model build- up, the respective weights are initialized and the input from the patient data set entered. Input are actual data patients suffering from prostate cancer. The whole network is now trained using Multiple Adaptive Linear Neuron (Madeline) techniques to recognize and associate patterns of input with desired output indicating the correct risk.

After this is run with internal processing in the network taking place and resultant output taking the value between -1(low PC risk) and 1(high PC risk).In some cases, the value is $<-1$ or $>1$ which is not relevant. Multiple runs of the model are now executed and a statistical curve indicating the values of PCa risk is plotted. If the curve indicates high occurrence of values closer to 1 then existence of prostate cancer is confirmed. The resultant data is not excreted but is feedback into the system so that a higher accuracy of the statistical curve applicable to patient belonging to similar data set is made possible.

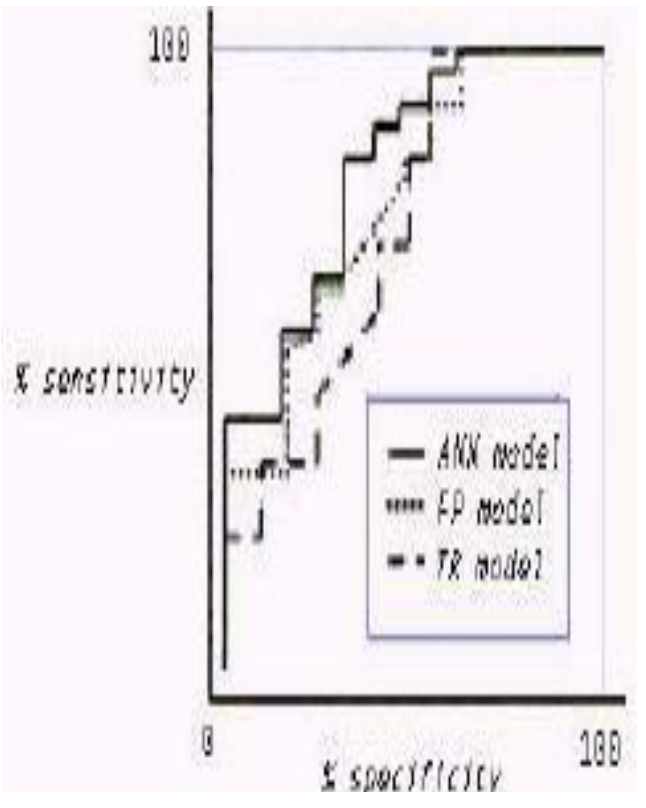

\section{CONCLUSION}

- Prostate cancer is the most common form of cancer among men according to statistical study result.

- Screening is a very rough estimate for cancer risk.

- $\quad$ Our proposed ANN model is far more efficient in predicting prostate cancer risk and reducing the no. of false positive test result.

- Our model is developed to aid or substitute the current diagnosis and prognosis methods.

- Our software test runs have shown that highly accurate ANN based on extending the MLP model can detect prostate cancer early and reduce unnecessary tissue sampling as compared to current methods such as tPSA\&fPSA

\section{Influencing factors}

- Larger no. of input variables from the patient data set can be supported.

- Interconnecting relationship between these variables and thereby formation of re-usable patterns can be established.

- The ability of the neural network to be trained time and again can be exploited thereby increasing accuracy each time.

- A deploy-ready easy to install software solution would be made available in the form of our PSRPSPANN Product which can install across various screening, hospital and research institutions

\section{REFERENCES}

1. Theoret MR, Ning YM, Zhang JJ, et al. The risks and benefits of 5a-reductase inhibitors for prostate-cancer prevention. N Engl J Med. 2011 Jun 15.

2. Antonarakis ES, Eisenberger MA. Expanding treatment options for metastatic prostate cancer. $N$ Engl $\mathrm{J}$ Med. 2011 May 26; 364:2055-2058.

3. Andriole GL, Crawford ED, Grubb RI 3rd, Buys SS, Chia D, Church TR, et al. Mortality results from a randomized prostate-cancer screening trial. $N$ Engl JMed.2009;360:1310-1319. 